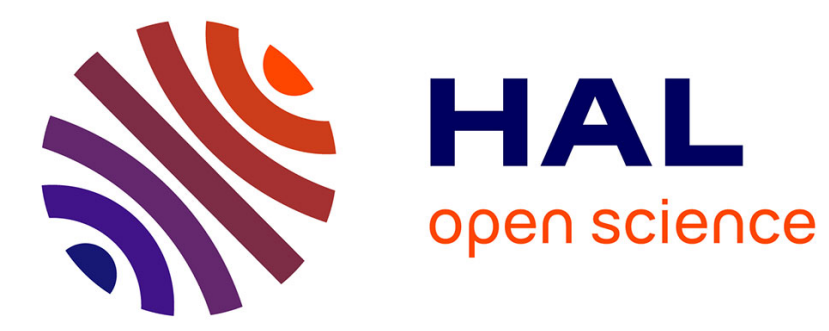

\title{
Évaluer la participation sociale de jeunes drépanocytaires. Recherche transdisciplinaire et adaptation des outils de recueil de données MHAVIE et MQE au contexte socioculturel de la Guadeloupe
}

Marie Cholley-Gomez, Sébastien Ruffié, Gaël Villoing, Normand Boucher, Patrick Fougeyrollas, Cholley-Gomez Marie, Ruffié Sébastien, Villoing Gaël, Bouchet Normand

\section{- To cite this version:}

Marie Cholley-Gomez, Sébastien Ruffié, Gaël Villoing, Normand Boucher, Patrick Fougeyrollas, et al.. Évaluer la participation sociale de jeunes drépanocytaires. Recherche transdisciplinaire et adaptation des outils de recueil de données MHAVIE et MQE au contexte socioculturel de la Guadeloupe. Aequitas Revue de développement humain, handicap et changement social, 2020, 26 (2), pp.43. 10.7202/1075205ar . hal-03404341

\author{
HAL Id: hal-03404341 \\ https://hal.science/hal-03404341
}

Submitted on 26 Oct 2021

HAL is a multi-disciplinary open access archive for the deposit and dissemination of scientific research documents, whether they are published or not. The documents may come from teaching and research institutions in France or abroad, or from public or private research centers.
L'archive ouverte pluridisciplinaire HAL, est destinée au dépôt et à la diffusion de documents scientifiques de niveau recherche, publiés ou non, émanant des établissements d'enseignement et de recherche français ou étrangers, des laboratoires publics ou privés. 
Evaluer la participation sociale de jeunes drépanocytaires. Recherche transdisciplinaire et adaptation des outils de recueil de données MHAVIE et MQE au contexte socioculturel de la Guadeloupe

\author{
Cholley-Gomez Marie*1, Ruffié Sébastien**1, Villoing Gaël**, Bouchet Normand*** et \\ Fougeyrollas Patrick*** \\ *Laboratoire IAPS, UFR STAPS, Université de Toulon \\ ** Laboratoire ACTES, UFR STAPS, Université des Antilles \\ *** Centre interdisciplinaire de recherche en réadaptation et intégration sociale (CIRRIS), \\ Université Laval, Québec, Canada \\ ${ }^{1}$ Les deux auteurs doivent être considérés tous les deux comme premiers auteurs pour leur \\ égale contribution à l'article.
}

Catégorie de l'article : savoirs académiques

Correspondant: sebastien.ruffie@univ-antilles.fr, UFR STAPS, Université des Antilles, Campus de Fouillole, BP 250, 97157 Pointe-A-Pitre cedex

Note des auteurs : Ce travail est issu d'un projet de recherche financé par la Fondation Maladies Rares et dirigé par l'équipe en sciences sociales de l'équipe de Recherche ACTES de l'Université des Antilles. L'Unité Transversale de la Drépanocytose et le Centre d'Investigation clinique du CHU de Pointe-A-Pitre ont été partenaires de ce projet qui a reçu un avis favorable du Comité de Protection des Personnes ${ }^{1}$ (CPP) Ouest VI en octobre 2018 puis en avril 2019 à la suite d'une modification.

Adresse du correspondant destiné aux lecteurs : sebastien.ruffie@univ-antilles.fr, UFR STAPS, Université des Antilles, Campus de Fouillole, BP 250, 97157 Pointe-A-Pitre cedex

\title{
Résumé :
}

Cet article présente un projet de recherche transdisciplinaire sur la participation sociale d'enfants et adolescents drépanocytaires guadeloupéens, présentant ou non des troubles neurocognitifs. L'ancrage théorique et méthodologique qui sous-tend ce projet est le modèle du MDH-PPH2 (Fougeyrollas, 2010 ; Fougeyrollas, Boucher \& Charrier, 2016) et ses outils de mesure (MHAVIE 4.0, Fougeyrollas, Noreau \& Lepage, 2014, et MQE 2.0, Fougeyrollas, Noreau, St-Michel, \& Boschen, 1999).

Dans un premier temps, les choix théoriques et méthodologiques sont justifiés, en particulier l'adaptation des échelles au contexte guadeloupéen, et à la maladie chronique caractérisant notre population principale ( $\mathrm{n}=102,6-16 \mathrm{ans})$. Un groupe contrôle de sujets sains $(\mathrm{n}=45)$, permet de vérifier que la participation sociale et la perception de l'environnement ne sont pas impactées par le contexte local, aucune étude n'existant à ce jour pour ce cadre socioculturel. L'axe qualitatif, constitué par des entretiens de type récits de vie, permet

${ }^{1}$ Le Comité de Protection des Personnes, ou CPP, évalue le respect des questions éthiques liées à un projet de recherche. Il s'agit de l'équivalent français des research ethics committees ou « Comités d'éthique de la recherche $»$. 
d'identifier les trajectoires de vie des drépanocytaires et de leur famille. Ce cadre temporel est pertinent au regard du MDH-PPH2, qui ajoute une perspective diachronique à la lecture initialement synchronique du modèle.

Enfin, la conclusion sera nourrie par la réflexivité et les considérations épistémologiques qu'implique un tel projet, particulièrement actuelles dans le champ de la santé (Trabal, Collinet \& Terral, 2017 ; Trabal, 2019).

Mots-clés : participation sociale, drépanocytose, processus de production du handicap, Guadeloupe, MHAVIE, MQE 
Social participation of young people with sickle cell disease living in Guadeloupe. Transdisciplinary research and local adaptation of the measurement scales LIFE-H and MQE

\section{Summary:}

This contribution describes a transdisciplinary research project studying the social participation of young people with sickle cell disease and eventually neuropsychological disorders, living in Guadeloupe. The theoretical and methodological framework leading this research is the second version of the Disability Creation Process (Fougeyrollas, 2010; Fougeyrollas, Boucher \& Charrier, 2016) and its measuring tools (LIFE-H4.0, Fougeyrollas, Noreau \& Lepage, 2014, and MQE 2.0, Fougeyrollas, Noreau, St-Michel, \& Boschen, 1999). Firstly, the theoretical and practical choices will be warranted: the cultural adaptation of the tools, regarding the specific local context, and regarding the chronic disease of our main study population $(\mathrm{N}=102$, between 6-16 years). A control group $(\mathrm{N}=45)$ allows us to investigate a possible effect of the cultural context on participation and on the perception of the environment, as no study has been conducted yet in such a context. The life trajectory or « carrier » of the young chronically ill and their close family can be highlighted by biographic narratives interviews. This timeframe is relevant in relation to the DCP-2, as it provides a diachronic perspective in addition to the initial synchronic description of the model. To conclude, we will focus attention on the epistemological issues implied by the crossdisciplinarity of such a research, a particularly topical issue in the field of health research (Trabal, Collinet \& Terral, 2017 ; Trabal, 2019).

Key-words: social participation, sickle cell disease, Disability Creation Process, Caribbean Studies, LIFE-H, MQE 


\section{INTRODUCTION}

La drépanocytose est une maladie de type autosomique récessive. Il s'agit de la pathologie génétique la plus répandue dans le monde, et la plus fréquente aux Antilles Françaises (Piel et al., 2013 ; Oudin-Doglioni et al., 2019). Elle est caractérisée par une falciformation des globules rouges (forme de «faucille», drepanon en grec), dont l'agglomération dans les vaisseaux rend difficile la circulation sanguine. L'obstruction conduit à une privation d'oxygène pour les organes et entraine des crises très douloureuses, principale conséquence de cette maladie (Parsh \& Kumar, 2012 ; Sundd, Gladwin \& Novelli, 2019). Ces crises, associées à une anémie chronique, et à des complications infectieuses parfois vitales, constituent les trois grands signes cliniques de la pathologie (Meremikwu \& Okomo, 2011).

En conséquence, les jeunes drépanocytaires, particulièrement sensibles au changement de température, à la déshydratation, aux risques infectieux, à l'effort et à l'altitude (HAS, 2014), font l'objet de préconisations médicales contraignant leurs activités sportives et de loisirs, dont la dimension sociale et culturelle est particulièrement forte. Bien que l'impact négatif de la drépanocytose sur la qualité de vie des enfants et adolescents drépanocytaires soit documenté (e.g., Graves, Hodge \& Jacob, 2016 ; Ludwig et al., 2018 ; Smaldone, Manwani \& Green, 2019), aucune étude n'a porté sur la participation sociale des drépanocytaires.

Dans le cadre d'un projet de recherche transdisciplinaire, impliquant des sciences biomédicales, neuropsychologiques et sociales, nous avons choisi de nous appuyer sur le Modèle du Développement Humain-Processus de Production du Handicap 2 (MDH-PPH2, Fougeyrollas, 2010 ; Fougeyrollas, Boucher \& Charrier, 2016), et ses outils, afin de comprendre comment la participation sociale d'enfants et adolescents drépanocytaires est impactée par la pathologie, leur vécu subjectif de celle-ci, et le traitement social dont elle fait l'objet, dans l'environnement spécifique de la Guadeloupe. Le projet vise ainsi à analyser la façon dont l'interaction entre des facteurs personnels et des facteurs environnementaux, physiques et sociaux, impactent la participation sociale de jeunes atteints d'un syndrome drépanocytaire majeur SS ou S $\beta 0$-thalassémie et pouvant être sujets à des troubles neuro-cognitifs. En effet, les drépanocytaires présentant une forme d'hémoglobinopathie de type SS ou SBthalassémie ${ }^{2}$ ont un taux important d'infarctus cérébraux dits « silencieux », c'est-à-dire non associés à des ${ }^{2}$ D'autres syndromes drépanocytaires majeurs présentent des formes hétérozygotes composites dans lesquelles
l'hémoglobine est associée à certaines hémoglobines anormales; formes S/C, S/D Punjab, S/Lepore, S/E, (EtienneJulan \& Saint-Martin, 2005), mais sont moins symptomatiques, et ne sont pas pris en compte dans ce projet. 
signes cliniques patents (Steen et al., 2005) et pouvant entrainer des troubles neurocognitifs (e.g., déficits de la fonction intellectuelle générale, des fonctions exécutives et attentionnelles, Steen et al., 2005 ; Berkelhammer et al., 2007).

Le projet de recherche s'inscrit donc dans une approche plurielle ; ainsi, outre les données médicales - recueillies par les médecins lors du suivi des patients - et les données neuropsychologiques - obtenues à partir de tests évaluant les fonctions cognitives - (i.e., facteurs personnels du PPH), nous nous sommes appuyés sur la mesure des habitudes de vie adaptée aux enfants (MHAVIE 4.0, Fougeyrollas, Noreau, \& Lepage, 2014) et la qualité perçue de l'environnement, (MQE 2.0, Fougeyrollas, Noreau, St-Michel, \& Boschen, 1999) pour évaluer la participation sociale des drépanocytaires.

L'utilisation de ces outils MHAVIE et MQE soulève cependant la question de leur adéquation à des contextes culturels particuliers (Boucher et al, 2017). N'ayant jamais été utilisés en Guadeloupe, nous avons entrepris, en tenant compte du contexte local et des caractéristiques de notre population d'étude, une démarche d'adaptation de ces instruments, particulièrement valorisée dans les travaux de recherche portant sur le handicap, à visée inclusive (Boucher et al., Ibid.). Cet article vise ainsi à présenter le protocole de recherche mis en place, ainsi qu'à expliquer et justifier la démarche d'adaptation culturelle de ces outils. Le «pluralisme» méthodologique et théorique de notre posture épistémologique nous semble apporter une contribution inédite à la littérature sur la participation sociale. De plus, celui-ci est particulièrement heuristique dans l'étude d'un objet complexe et multiple tel que la drépanocytose, situé à l'intersection de divers regards disciplinaires. Une maladie qui fut longtemps l'objet, au même titre que les hémoglobinopathies, d'une biomédicalisation importante (Pruneau et al., 2009 ; Dyson, 2019) et d'un investissement tardif par les travaux socio-anthropologiques. Les problématiques et les réflexions soulevées par une telle démarche seront discutées en conclusion, sous la forme d'une ébauche qui a été poursuivie et approfondie dans une récente contribution (Ruffié et al., 2020).

\section{CADRE THEORIQUE}

La participation sociale des drépanocytaires : une approche systémique par le modèle du

\section{Processus de Production du Handicap... et de la maladie}

La drépanocytose peut s'inscrire dans la Classification Internationale du Fonctionnement, du handicap et de la santé (CIF, OMS, 2001) favorisant la considération de sa complexité. Cet 
outil de classification, originellement orienté vers les « conséquences de la maladie », vise la description des « composantes de la santé », et adopte ainsi une posture neutre par rapport à l'étiologie. Il propose également de considérer, dans l'étude des déterminants de santé, une liste de facteurs environnementaux permettant la description du contexte de vie de chaque individu. Une telle approche est nécessaire car la drépanocytose a été, dans une large mesure, étudiée à partir d'une perspective médicale ou biomédicale, reflet de la faible pénétration des questions sociales ou politiques dans les travaux sur les hémoglobinopathies (Atkin \& Ahmad, 2001 ; Bediako \& Moffitt, 2011). Plus généralement, les problématiques liées à la construction sociale de cette maladie, à sa racialisation et ses effets sur l'expérience des malades n'ont que récemment émergé dans les travaux scientifiques (Lainé \& Tchernia, 2010 ; Dyson, 2019).

L'ensemble des dimensions sociodémographiques et identitaires en jeu, ainsi que les liens entre facteurs personnels et environnementaux, dans leurs effets sur la participation sociale, doivent être envisagés afin de saisir la complexité des situations de handicap que les drépanocytaires peuvent vivre. Si les environnements médicaux et familiaux sont souvent protecteurs, voire surprotecteurs, par contraste les environnements sociaux et culturels produisent une forte stigmatisation (Bougerol, 1994 ; Poku, Caress \& Kirk, 2020 ; pour une revue systématique, se reporter à Bulgin et al., 2018). La diversité des environnements et leur articulation complexifient les actions à initier pour favoriser la participation des drépanocytaires dans une visée inclusive. Mais au-delà des effets environnementaux, les aspects personnels (e.g., vécu de la maladie chronique, capacités cognitives, image de soi) sont également générateurs de difficultés et de limitation de la participation sociale (Dumont, 2003 ; Larivière, 2008). On perçoit dès lors l'effet cumulatif des difficultés d'insertion et de participation à la société auxquelles s'ajoutent les difficultés neuro-cognitives, nécessitant une approche multifactorielle de la drépanocytose : comme un fait biologique, mais aussi comme phénomène social.

À ce jour, aucune étude sur la participation sociale des drépanocytaires ne procède du modèle théorique interactionniste proposé par le PPH. Pourtant, les effets de la déficience physique, des limitations cognitives ou psychologiques doivent être mis en relation avec les environnements afin de contextualiser les conséquences de la maladie dans la réalisation des habitudes de vie et la participation sociale des enfants et adolescents drépanocytaires. Le choix d'une approche transdisciplinaire est rendu pertinent par la définition du handicap ; à l'origine médicale, elle a acquis une orientation sociopolitique et anthropologique (Szymanski et al., 1990 ; Fougeyrollas, et al., 1998). Dans cette perspective, la participation est ainsi le produit de la rencontre entre «des personnes différentes (ayant des caractéristiques fonctionnelles 
limitantes, les singularisant) et un contexte plus ou moins accueillant » (Fougeyrollas, cité dans Boukala, 2009 : 167). La situation de handicap n'est donc pas une caractéristique, un élément intrinsèque de l'individu, mais elle est conjoncturelle et relative (Fougeyrollas et al., 2015 ; Ravaud \& Fougeyrollas, 2005 ; Ravaud et al., 2002). En considérant le processus d'interaction complexe entre déficience, incapacité, identité et environnement physique et socioculturel, la définition de la situation de handicap, proposée par l'approche du $\mathrm{PPH}$, permet de rendre compte de la perte contextualisée (et plus ou moins momentanée) d'autonomie en considérant les facilitateurs, et les freins ou obstacles à la participation sociale.

Si le modèle du PPH fut principalement utilisé dans la compréhension de l'expérience du handicap des personnes ayant des incapacités de divers ordres (moteur, cognitif, sensoriel), il est d'une grande pertinence pour l'étude de la maladie chronique, comme en attestent les travaux de Ferez et al. (2015), et de Lacroix, Boucher et Villeneuve (2016) qui ont démontré l'intérêt d'une telle modélisation pour saisir respectivement l'expérience du VIH et du vécu de la sclérose en plaques. La notion de participation sociale, au travers de la réalisation d'habitudes de vie, permet d'établir des liens avec d'autres groupes susceptibles d'éprouver des difficultés d'intégration ou des limitations de leur participation, en considérant les caractéristiques spécifiques des individus ayant des incapacités (Doré, 2007 ; Fougeyrollas et al., 2015). Plus généralement, Fougeyrollas (2010) invite les chercheurs à ne pas circonscrire l'utilisation de son modèle à la compréhension et à l'expérience spécifique du handicap, mais de l'appliquer aussi à la maladie chronique : il concerne tous les individus pour lesquels la matrice normative éco-sociale est source de discrimination ou de privilège dans leur participation sociale, en fonction de l'interprétation donnée à une différence corporelle, comportementale ou esthétique (Fougeyrollas \& Blouin, 1989). Ainsi, les politiques et actions d'intégration visent un grand nombre de «différences» et de déficiences, physiques ou mentales, regroupées par Fougeyrollas $(1983,2010)$ sous l'appellation de « corps différents ». Car au-delà, c'est bien dans une perspective inclusive et pragmatique que les travaux relevant du PPH doivent s'inscrire, car exercer sa participation sociale, signifie exercer ses droits ${ }^{3}$. Le modèle choisi en appui de ce travail, le Processus de Production du Handicap (dans sa dernière version MDHPPH2, Fougeyrollas, Boucher \& Charrier, 2016) est consensuellement approuvé dans le champ

\footnotetext{
${ }^{3}$ Comme le précise Fougeyrollas (dans Boukala, 2009, p.167, nous soulignons) : « Participer socialement signifie avoir la possibilité de jouer ses rôles de citoyen dans la vie de tous les jours. Et j'insiste sur la distinction entre être capable de et puis avoir la possibilité de. Préparer les repas, élever nos enfants, travailler, avoir des loisirs et des relations sociales sont autant d'activités et de rôles sociaux que réunit l'expression participation sociale. Elle correspond donc à la fois aux attentes d'une personne et à celles du contexte dans laquelle elle évolue pour pouvoir être considérée comme un homme ou une femme ordinaire ».
} 
de la réadaptation au Québec (Dumont, 2003) en raison de la clarté et de la cohérence de sa segmentation conceptuelle (Noreau, 2005).

\section{METHODE}

Le projet de recherche « Sociodrep » : drépanocytose, troubles cognitifs et participation sociale

C'est à partir de l'ancrage théorique et méthodologique au PPH que s'est élaboré notre projet de recherche transdisciplinaire ${ }^{4}$, mené conjointement par des équipes en sciences sociales $^{5}$ et l'Unité Transversale de la Drépanocytose, également appelé Centre de la Drépanocytose. La drépanocytose fait l'objet d'un programme global de prise en charge au sein d'un centre intégré rattaché au CHU de Pointe-À-Pitre, constituant la première unité de ce type sur le territoire français (Bibrac \& Etienne-Julan, 2004). Ce centre de référence initie une approche complexe et plurielle de prise en charge par l'articulation de l'accompagnement médical, psychologique et social des patients et la mise en place d'actions de prévention déployées vers la société antillaise. Des médecins pédiatres, des neuropsychologues et des informaticiens du Centre d'Investigation Clinique du CHU participent donc activement à cette recherche.

Un schéma ci-après (Figure 1) présente la méthodologie générale de ce projet et les deux phases de recueil : la démarche exploratoire visant à adapter les outils utilisés, puis le recueil des données de l'étude. Le projet couvrait initialement une période de deux ans ${ }^{6}$. La cohorte initiale de sujets est constituée de jeunes drépanocytaires ( $\mathrm{n}=102$, de 6 à 16 ans, 49 filles, 53 garçons), porteurs d'un Syndrome Drépanocytaire Majeur SS ou S $\beta 0$-thalassémie et pouvant présenter ou non des troubles neurocognitifs. Tous les sujets sont suivis par le Centre de Référence de la Drépanocytose (CHU de Pointe-À-Pitre et de Basse-Terre, CHBT).

Figure 1. Représentation schématique des phases d'adaptation des outils et de recueil des données

\footnotetext{
${ }^{4}$ Ce projet fait l'objet d'un financement par la Fondation Maladies Rares et par le Centre Hospitalier Universitaire (CHU) de Pointe-À-Pitre.

${ }^{5}$ Le projet est porté par l'équipe de recherche ACTES de l'UFR STAPS de l'Université des Antilles (2 enseignantschercheurs en sociologie et 2 post-doctorants en psychologie et sociologie pour le recueil des données). L'équipe médicale de l'Unité Transversale de la Drépanocytose mobilisée sur le projet est constituée de médecins pédiatres, infirmières et psychologues. Le CIC (Centre d'Investigation Clinique) est également mobilisé, un biostatisticien, data manager gérant le stockage des données. Le projet est mené en collaboration avec l'équipe du CIRRIS, de l’Université Laval, Québec, et l'équipe SantesiH, de l’Université de Montpellier.

${ }^{6} \mathrm{Du}$ fait de la crise sanitaire, le rendu du rapport final aux financeurs est reporté à 2021.
} 


\begin{tabular}{|c|c|}
\hline $\begin{array}{c}\text { Phase } 1 \text { : adaptation des outils } \\
\text { Mhavie et MQE } \\
\text { (avril-septembre 2018) }\end{array}$ & $\begin{array}{l}\text { Phase } 2 \text { : recueil de données (novembre 2018-juin } \\
\text { 2019) }\end{array}$ \\
\hline $\begin{array}{l}\text { 1) Revue de littérature (participation } \\
\text { sociale, drépanocytose, contexte } \\
\text { guadeloupéen) } \\
\text { 2) Enquête exploratoire : échanges } \\
\text { avec les acteurs de terrain (e.g., } \\
\text { médecins, familles de malades membres } \\
\text { d'association) ; Echanges réguliers avec } \\
\text { les concepteurs des outils pour } \\
\text { validation et ajustement.s éventuel.s } \\
\text { 3) Passation-test des outils Mhavie \& } \\
\text { MQE pour vérifier la compréhension et } \\
\text { pertinence des items (N=4 } \\
\text { drépanocytaires } \mathrm{SC}, \mathrm{M}=8.75, \mathrm{ET}= \\
\text { 5.12) }\end{array}$ & $\begin{array}{c}\text { Axe biomédical : données de suivi (e.g bilan } \\
\text { hématologique, vasculopathie cérébrale, crises } \\
\text { vaso-occlusives, infections) cohorte } \\
\text { drépanocytaire : N }=102 \text {. } \\
\text { Axe neuropsychologique : évaluation de } \\
\text { l'efficience intellectuelle globale (WISC-V, } \\
\text { Wechsler, 2005), et des fonctions exécutives } \\
\text { (BRIEF, Gioia et al., 2000, NEPSY II, } \\
\text { Korkman et al., 2007, et figure de Rey Rey, } \\
1959) . \mathrm{N}=76, \text { Mâge }=11, \mathrm{ET}=3 \text {. } \\
\text { Axe Sciences sociales : } 1 \text { ) Mhavie }+M Q E \\
\text { (drépanocytaires : } 86 \text { répondants, analyses } \\
\text { finales : } \mathrm{N}=81, \mathrm{Ma} g \mathrm{ge}=11.22+/-3.22 ; \\
\text { Groupe contrôle : } \mathrm{N}=45, \mathrm{M}=10.55+/-2.93 \text { ) } \\
\text { 2) Entretiens biographiques }(\mathrm{N}=40 \text {, dont } 2 \text { hors } \\
\text { cohorte) }\end{array}$ \\
\hline
\end{tabular}

\section{Phase d'adaptation des échelles MHAVIE \& MQE}

\section{1. Présentation des outils}

La Mesure des Habitudes de Vie (MHAVIE 4.0 pour enfants et adolescents, Fougeyrollas, Noreau \& Lepage, 2014) et la Mesure de la Qualité de l'Environnement (MQE 2.0, Fougeyrollas, Noreau, St-Michel, \& Boschen, 1999) sont des échelles de mesure validées et présentant de très bonnes qualités psychométriques?

La MHAVIE est un outil construit sur la base de la segmentation conceptuelle du MDHPPH. Elle évalue la perception de la personne face à la réalisation de ses habitudes de vie, désignant une activité courante ou un rôle social valorisé par la personne ou son contexte socioculturel, en fonction de ses caractéristiques personnelles (âge, sexe, identité) et assurant l'épanouissement d'un individu tout au long de sa vie (Fougeyrollas, 1998). Cet outil est conçu pour une auto-administration, mais peut, le cas échéant, si l'incapacité le nécessite, être complété par un tiers, proche significatif du jeune (Fougeyrollas et al., 2014). Les habitudes de vie sont déclinées en douze catégories d'activités courantes et de rôles sociaux, appréciées à partir d'une échelle de mesure allant de la « situation de participation sociale optimale » à la «situation de handicap incomplète », résultant de l'interaction entre facteurs personnels et facteurs environnementaux (obstacles et facilitateurs) (Fougeyrollas \& Charrier, 2013,

\footnotetext{
${ }^{7}$ Pour la consistance interne de la MHAVIE-enfants, $0.82<\mathrm{ICC}<0.9$; fidélité intra-juges : $\alpha>0.90$, Bedell \& Coster, 2008 ; Fougeyrollas et al., 2014 ; et, pour la MQE, entre $56 \%$ et $85 \%$ des items ont démontré des scores de concordance de $60 \%$ et plus, indiquant une fidélité modérée à élevée (Fougeyrollas et al., 2014).
} 
Fougeyrollas et al., 2016). Le participant indique le degré de difficulté perçu (sans ou avec peu de difficultés, avec une certaine difficulté, avec beaucoup de difficultés). Une mesure de la satisfaction est également proposée pour chaque item et, bien que ne contribuant pas au calcul du score global de participation, elle constitue une dimension importante de la MHAVIE et peut faire l'objet d'une analyse distincte (Fougeyrollas, Noreau \& Lepage, 2014 ; Boucher et al., 2015).

Si dans la littérature, certains auteurs distinguent activités courantes (i.e., de la vie quotidienne et domestique) et rôles sociaux, ces derniers étant supposés supérieurs dans leur contribution à la participation sociale (Boisvert, 2006 ; Lachapelle, 2003), la perspective anthropologique défendue par le MDH-PPH, postule le caractère socioculturellement construit de toutes les activités humaines (Bourdieu, 1980 ; Mauss, 1936), et n'appelle pas à une telle hiérarchie.

La MQE évalue l'influence des facteurs environnementaux, qui renvoient aux dimensions physiques ou sociales et déterminent l'organisation et le contexte d'une société (Fougeyrollas, 1998). L'instrument est composé d'items formulés à partir de la nomenclature des facteurs environnementaux issus de la classification du modèle de Processus de Production du Handicap (Fougeyrollas et al., 1998). Ces facteurs sont politico-économiques et socio-culturels ; ceux d'ordre physique renvoient à la nature et à l'aménagement. Au total, la MQE se compose de 6 facteurs :1) Support et attitudes de la famille et des amis ; 2) Revenu, emploi, et sécurité du revenu ; 3) Services gouvernementaux et publics ; 4) Environnement physique et accessibilité ; 5) Technologie et 6) Égalité des chances et orientations politiques.

L'influence perçue de chaque facteur est mesurée à l'aide d'une échelle allant du facilitateur majeur (+3) à l'obstacle complet (-3). Un facteur peut n'avoir aucune influence (0) ou ne pas s'appliquer à la situation de vie de la personne. Un facilitateur majeur se définit comme un facteur qui compense entièrement les déficiences ou incapacités permettant ainsi à la personne de réaliser pleinement son habitude de vie sans contrainte ni difficulté. Lorsqu'un facteur environnemental entrave la réalisation des habitudes de vie, en interaction avec les caractéristiques personnelles, il constitue un obstacle qui occasionne une situation de handicap (Fougeyrollas et al., 2015).

\section{2. Etapes de la démarche d'adaptation}

Les consignes méthodologiques portant sur la MHAVIE et la MQE indiquent qu'il est possible de modifier certains items ou énoncés en fonction de la réalité culturelle dans laquelle est utilisé l'outil, sans modifier les catégories des instruments et les échelles de réponse, ce qui porterait potentiellement atteinte à sa structure et à sa validité (Boucher et al., 2017). Dans le 
cadre de notre recherche, il convenait d'adapter ces outils à l'expérience de la drépanocytose ainsi qu'au contexte culturel antillais. À titre d'exemple, dans la littérature foisonnante portant sur la qualité de vie, de nombreux auteurs ont adapté l'échelle d'évaluation de la qualité de vie pour rendre compte des spécificités des populations concernées par les travaux ${ }^{8}$.

D'un point de vue méthodologique, plusieurs étapes ont présidé à l'adaptation des outils. Dans un premier temps, diverses sources nous ont permis, dans le cadre d'une démarche exploratoire, d'identifier les dimensions pertinentes dans la réalisation des habitudes de vie et des rôles sociaux (i.e., la participation sociale) pour une population de jeunes drépanocytaires (6-16 ans) vivant en Guadeloupe. Pour rappel, si la littérature apporte des éléments relatifs à la qualité de vie des drépanocytaires (e.g., Thomas \& Taylor, 2002 ; Benjamin, 2008 ; Adeyemo et al., 2015 ; Matos, Malheiros \& Matos, 2016) aucune recherche n'a à ce jour porté sur l'évaluation de la participation sociale, et de ses déterminants, chez des sujets drépanocytaires. De tels travaux ont été conduits dans le champ du handicap, des traumatismes cérébraux, ou encore de la déficience intellectuelle et des troubles mentaux (Lepage, Noreau \& Bernard, 1998 ; Paré et al., 2000 ; Desrosiers et al., 2002 ; Noreau et al., 2004 ; Donkervoort et al., 2007 ; Grandisson, Thétrault \& Freeman, 2010 ; Larivière, 2012 ; Fougeyrollas et al., 2014).

Notre démarche d'adaptation fut structurée temporellement comme suit :

a) Nous avons constitué une revue de la littérature portant sur la participation sociale, mais également sur la drépanocytose et le contexte guadeloupéen afin d'identifier certaines inadéquations des questionnaires (i.e., manque ou inadaptation de certains items) liées à la pathologie et aux spécificités culturelles.

Par exemple, sur les facteurs climatiques présentés dans la MQE, nous avons opéré des modifications pour rendre compte des caractéristiques spécifiques du climat local. En effet, on distingue en Guadeloupe deux saisons fondamentales : une saison sèche, et marquée par un flux d'alizés (vents) plus fréquents, qualifiée de « carême », et une saison chaude et humide, du fait des averses plus nombreuses et intenses, marquée par la possible présence de cyclones, appelée «l'hivernage ». Ces éléments ont donc été intégrés aux items portant sur la perception des éléments naturels. Au sein de la catégorie « Soins personnels et de santé » de la MHAVIE, nous avons intégré la gestion de la crise sous la forme de l’item suivant : « Gérer la situation en cas

\footnotetext{
${ }^{8}$ Citons le VQ11, la HR-QOL (Health-Related Quality of Life) pour sclérosés en plaques (Vickrey et al., 1995); le questionnaire de qualité de vie spécifique à la BPCO (Ninot et al., 2010); l'échelle de qualité de vie SF-12 chez les personnes vivant avec le VIH au Cameroun (Préau et al., 2006) ; la WHOQOL-AGE, mesure de la qualité de vie chez les personnes âgées (Caballero et al., 2013); ou encore la QOL-MV, mesure de la qualité de vie chez des patients en soin intensifs placés sous respiration assistée mécaniquement (Quality of Life Questionnaire for Mechanically Ventilated Intensive Care Unit Patients, Pandian et al., 2015).
} 
de crise douloureuse (s'allonger, demander de l'aide, appeler les secours...) ». En effet, comme indiqué précédemment, la drépanocytose est caractérisée par la survenue de crises très douloureuses, pouvant conduire à des hospitalisations en fonction de leurs intensités, et pouvant être à l'origine de troubles psycho-sociaux chez les jeunes drépanocytaires (Carpentier, Elkin \& Starnes, 2009 ; Benton et al., 2011). Ces épisodes douloureux, manifestations cliniques de crises vaso-occlusives (obstruction vasculaire), sont en effet imprévisibles en intensité, fréquence et durée (Galactéros, 2005; Sundd, Gladwin \& Novelli, 2019) et surviennent davantage durant l'enfance et l'adolescence qu'à l'âge adulte.

Précisons que certains ajustements intègrent ainsi à la fois des adaptations au contexte de la drépanocytose et au contexte antillais : par exemple, la baignade en mer ou rivière est à la fois un loisir social important en Guadeloupe (Pruneau et al., 2008), mais elle est aussi une activité à fort risque pour les drépanocytaires, puisqu'elle est susceptible d'entrainer une crise vasoocclusive douloureuse du fait de la différence de température éprouvée par le corps.

b) A la suite de ce travail préalable, nous avons entrepris un travail d'échange avec les concepteurs des échelles et du modèle du PPH (équipe du CIRRIS, Québec) ainsi qu'avec les acteurs de terrain pour élaborer nos premières modifications. Ces personnes ressources furent composées ${ }^{9}$ :

i. Des praticiens du champ médical au contact de la pathologie (médecins pédiatres et psychologues du centre de référence caribéen de la drépanocytose) ;

ii. Membres d'associations de malades ou de parents de malades présents sur le territoire et impliqués dans le projet (Guadeloupe Espoir, Drépano Debout et Drép'Agir) ;

iii. Issues du Rectorat de Guadeloupe, confronté aux difficultés singulières de ce public, notamment en ce qui concerne les diverses installations pédagogiques (présence ou absence de climatiseurs), les cours d'EPS, ainsi que leur accompagnement scolaire $\left(\mathrm{AVSI}^{10}\right)$;

Ces diverses sources d'informations nous ont permis d'ajuster nos transformations, et d'opérer lorsque nécessaire de nouvelles modifications. À chaque étape, nous informions les concepteurs et nous nous assurions de leur retour positif afin de maintenir ces ajustements.

\footnotetext{
${ }^{9}$ Pour une démarche similaire dans la validité de contenu de la MS-QUOL54, se reporter à Vickrey et al, 1995.

${ }^{10}$ Dispositif AVSI (Auxiliaire de Vie Scolaire Individuel) : personne qui aide à l'inclusion scolaire des élèves présentant des difficultés ou en situation de handicap. L'aide est attribuée par la commission des droits et de l'autonomie des personnes handicapées au sein des MDPH (Maisons Départementales des Personnes Handicapées) qui se prononce au regard d'une évaluation de la situation scolaire de l'élève.
} 
c) Enfin, une fois la version finale des outils mise en place, deux chercheurs en Sciences Sociales ont réalisé, dans le cadre d'une démarche exploratoire, une passation avec quatre enfants ( 2 garçons, 2 filles), âgés de $7,8,14$ et 16 ans $(\mathrm{M}=8.75$, ET $=5.12)$ et drépanocytaires $\mathrm{SC}^{11}$. Ils se sont ainsi assurés de la bonne compréhension de l'outil par le jeune et l'accompagnant (dans ce cas, la mère), du temps de passation nécessaire (estimé entre $1 \mathrm{~h}$ et $1 \mathrm{~h} 30$ ) et de la pertinence des items et du contenu de chaque instrument.

d) La dernière étape de modification se concrétisa par la soumission des différentes modifications et des outils aux membres du RIPPH concepteurs de la MHAVIE et de la MQE afin d'obtenir leur avis puis accord pour le démarrage de l'étude et la passation finale.

\section{I.3. Modifications et structure finale de la MHAVIE}

Suivant les recommandations méthodologiques liées à d'éventuelles modifications des outils du PPH (Boucher et al., 2017), qui incitent à les ajuster au contexte local et culturel, nous n'avons pas, dans un souci de maintien des qualités métrologiques de ces derniers, altéré la structure de la MHAVIE. Ainsi sont conservées ses dimensions et sous-dimensions (catégories d'activités quotidiennes, et de rôles sociaux), ainsi que les modalités de réponse (réalisation ou non de l'activité, type d'aide requis, satisfaction éprouvée).

Pour chaque énoncé, le répondant indique ainsi le niveau de réalisation (réalisé, que ce soit par la personne elle-même, ou par substitution ; non réalisé ; ne s'applique pas), le type d'aide requis (aide technique : par exemple une bouteille d'eau que le jeune garde constamment avec lui ; aménagement, par exemple, du temps supplémentaire pour accomplir l'activité ; aide humaine, sur une échelle à 4 points : aucune, légère, importante ou complète), et enfin, le niveau de difficulté perçu (trois modalités). Le répondant indique enfin le niveau de satisfaction à l'égard de chaque habitude de vie sur une échelle en 4 points (de «très satisfait » à «très insatisfait »). La satisfaction du répondant est une dimension importante de la MHAVIE, et ce, même si le résultat n'est pas inclus dans le calcul du score global de participation sociale (Fougeyrollas, Noreau \& Lepage, 2014). Ce score d'accomplissement global, ou pour chaque habitude de vie et catégorie, permet de situer le degré de participation sociale du sujet, sur un continuum de 0 à 9 , où 0 représente une situation de handicap totale (i.e., absence de réalisation
Commenté [CB1]: S'agit-il de la forme SC de la maladie ? Auquel cas correspond-elle à l'une des deux formes (Syndrome Drépanocytaire Majeur SS ou S $\beta 0$-thalassémie ) dont il est dit en $p .7$ que Ice sont les deux formes dont sont porteurs les enfants de la cohorte étudiée.

\footnotetext{
${ }^{11}$ La forme hétérozygote SC est la forme la plus répandue après la forme drépanocytaire SS. Elle est caractérisée par la présence de deux hémoglobinoses $\mathrm{S}$ et $\mathrm{C}$ sous leur forme hétérozygote. Les caractéristiques physiopathologiques de ces formes sont toutefois comparables, l'hémoglobine $\mathrm{HbC}$ favorise également la falciformation. La forme $\mathrm{S} \beta 0$-thalassémie est, quant à elle, une forme composite plus rare.
} 
des habitudes de vie du fait de l'interaction entre des facteurs personnels et environnementaux), et 9 une participation optimale (réalisation sans aide ni difficulté).

En outre, nous n'étions pas en mesure d'assurer la fiabilité test-retest des outils, en raison de contraintes matérielles (durée du projet, obligations liées au CPP (Comité de Protection des Personnes) et au recueil de données sur une population fragile ne permettant pas de réaliser une passation en Temps 1 - Temps $2^{12}$ ). Cependant, nous aurons la capacité de fournir des éléments quant aux liens (de corrélation et de prédiction) entre les données évaluant la participation sociale obtenues grâce aux questionnaires et celles rendant compte de la sévérité de la maladie présentes dans le dossier médical des jeunes (intensité et nombre de crises subies, d'infections, d'hospitalisations ${ }^{13}$ ) et la présence de troubles neuro-cognitifs grâce aux tests psychométriques réalisés (i.e., fonctions exécutives et attentionnelles). Ces résultats pourront contribuer à assoir la pertinence de nos instruments ${ }^{14}$.

Les modifications apportées ont donc consisté à fusionner, modifier, ajouter ou supprimer certains items. Les figures 2 et 3 ci-après présentent un exemple d'adaptation pour les catégories Déplacements et Vie associative et spirituelle ${ }^{15}$.

Figure 2. Catégorie « Déplacements » de la MHAVIE, version originale à gauche, modifiée à droite.

\section{Déplacements}

Entrer et sortir de sa résidence

Se déplacer à l'intérieur de sa résidence

Se déplacer sur le terrain de sa

résidence (cour, parterre...)

Se déplacer dans la rue ou sur le trottoir

Traverser la rue à une intersection

${ }^{12}$ Les jeunes sont souvent sollicités pour des questionnaires MHAVIE et MQE (temps de pass d'une autre passation de la MHAVIE et la MQE al les jeunes et les familles, mais également diffic entrainé certainement le refus du CPP obligatoire ${ }^{13}$ Par exemple, en fonction des relevés sanguins, transcranien (vasculopathie); ces informations $\mathrm{q}$ hématologiques à l'état de base : le degré de l' réticulocytes, $\mathrm{LDH}$ ), le taux de leucocytes et de ne fœtale.

${ }^{14}$ Vickrey et al., (1995) ont ainsi procédé pour jus de vie sur une population de sclérosés en plaques.

${ }^{15}$ Le volume limité du manuscrit ne nous perme lecteur est invité à écrire aux co-premiers auteur toute demande de précision ou d'information com

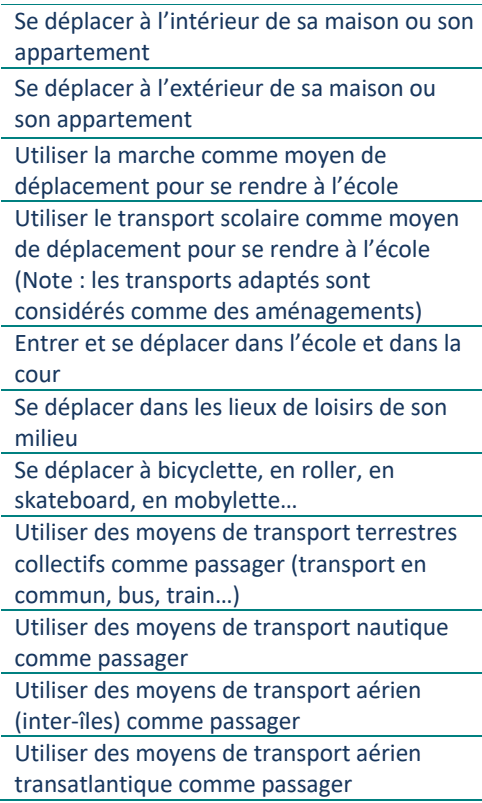


Se déplacer sur une surface glissante ou

inégale (neige, glace, herbe, gravier...)

Utiliser la marche ou le transport

scolaire comme moyen de déplacement

pour se rendre à l'école

(Note : les transports adaptés sont

considérés comme des aménagements)

Entrer et se déplacer dans l'école et

dans la cour

Se déplacer dans les lieux de loisirs de

son milieu

Entrer et se déplacer dans les

commerces et les services (restaurant,

épicerie, cliniques médicales...)

Se déplacer à bicyclette

Utiliser une voiture comme passager

(monter, descendre, s'attacher...)

Utiliser des moyens de transport comme passager (transport en commun, taxi...)

Utiliser la marche ou le transport

scolaire comme moyen de déplacement

pour se rendre à l'école

Note : les transports adaptés sont

considérés comme des aménagements

Entrer et se déplacer dans l'école et

dans la cour

Se déplacer dans les lieux de loisirs de

son milieu

Entrer et se déplacer dans les

commerces et les services (restaurant,

épicerie, cliniques médicales...)

Se déplacer à bicyclette

Utiliser une voiture comme passager

(monter, descendre, s'attacher...)

Utiliser des moyens de transport comme

passager (transport en commun, taxi...)

Figure 4. Catégorie « Déplacements » de la MHAVIE, version originale à gauche, modifiée à droite.

Vie associative et spirituelle

Participer à titre de membre à des associations étudiantes (conseil

d'école, conseil de classe, comité

parascolaire...)

Participer à titre de membre à des

associations de loisirs (scouts,

club ...)

Participer aux activités de groupes

d'entraide ou d'intérêts (droits de la

personne, écologie...)

Participer comme membre à des associations scolaires (conseil d'école, conseil de classe, comité parascolaire...)

Participer comme membre à des associations de

loisirs (groupes carnavalesques ...)

Participer à des activités reliées à des pratiques

religieuses ou spirituelles (au domicile, à l'église

ou autres lieux de culte, les cérémonies...) 


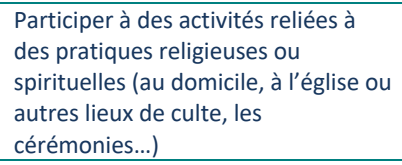

Le tableau 1 ci-après donne une vision générale et quantitative des modifications opérées sur la MHAVIE et indique, pour chaque activité quotidienne ou rôle social, le nombre d'items initial, et le nombre d'items contenu dans l'échelle finale.

Tableau 1. Nombre d'items par domaine de la version originale et modifiée de la MHAVIE

\begin{tabular}{lcclrr}
\hline Activités quotidiennes & \multicolumn{2}{l}{ nb items } & Rôles sociaux & \multicolumn{2}{l}{ nb items } \\
\hline Communication & $9^{*}$ & $9^{* *}$ & Responsabilités & $8^{*}$ & $7^{* *}$ \\
\hline Déplacements & $13^{*}$ & $11^{* *}$ & Relations interpersonnelles & $8^{*}$ & $7^{* *}$ \\
\hline Nutrition & $6^{*}$ & $3^{* *}$ & Vie associative et spirituelle & $4^{*}$ & $3^{* *}$ \\
\hline Condition physique & $5^{*}$ & $4^{* *}$ & Education & $7^{*}$ & $6^{* *}$ \\
et bien-être psychologique & & & & & \\
\hline Soins personnels et de santé & $10^{*}$ & $6^{* *}$ & Travail & $3^{*}$ \\
\hline Habitation & & & & & \\
\hline
\end{tabular}

\section{I.4. Modifications et structure finale de la $M Q E$}

Dans la MQE, certains facteurs ont été supprimés du fait de leur faible pertinence pour notre contexte et notre population. Au sein des facteurs sociaux, les catégories «services commerciaux » et « services juridiques » n’ont pas été conservées dans l'outil final ; parmi les facteurs physiques, les catégories «accessibilité physique», «objets», « technologie» et «aides techniques » ont été supprimées. Le facteur «marché du travail»a été requalifié en « milieu professionnel et de stage », mieux adapté à l'âge de notre population. À nouveau, la structure des modalités de réponse n'a pas été modifiée. L'échelle de perception des facteurs environnementaux est ainsi conservée sous la forme d'une échelle en 7 points, allant de l'obstacle complet (-3) au facilitateur majeur $(+3)$. Pour le calcul de scores, il est possible de recoder les réponses (données sur une échelle de Likert en 7 points) en trois catégories : facteur constituant un obstacle, un facilitateur, ou sans influence (Boucher et al., 2015).

Du fait de la complexité de certains items (e.g. au sein des systèmes politiques ou services socio-sanitaires), nous avons considéré que la passation de certaines catégories de 
l'outil nécessitait la présence et la réponse d'un parent ou d'un proche du jeune, lorsque l'âge de ce dernier rend difficile la compréhension des énoncés. Les figures 4 et 5 ci-après présentent un exemple d'adaptation pour le facteur environnemental Services socio-sanitaires, et Services Educatifs.

Figure 4. Catégorie « Services socio-sanitaires » de la MQE originale (à gauche) et finale (à droite).

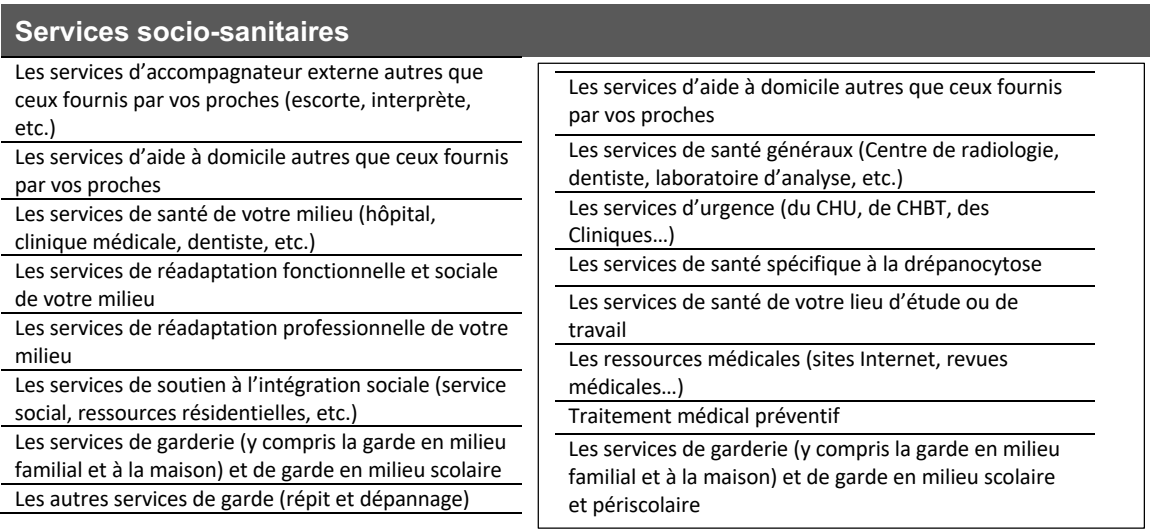

Figure 5. Catégorie « Services éducatifs » de la MQE originale (à gauche) et finale (à droite).

\section{Services éducatifs}

Les services éducatifs de votre milieu (primaire, secondaire, collégial, universitaire, éducation des adultes, formation professionnelle, etc.)

L'accès aux prêts et bourses aux étudiants

Les autres services de votre milieu scolaire (activités parascolaires, cafétéria, services aux étudiants, etc.)

Les services de transport scolaire

\begin{tabular}{l}
\hline Les services éducatifs (école, collège, lycée) \\
\hline Les services d'orientation et de recherche de \\
formation ou de professionnalisation (critères d'entrée \\
en formation ou les tests de sélection...) \\
\hline Les autres services de votre milieu scolaire (activités \\
parascolaires, UNSS, USEP, etc.) \\
\hline Les services de votre milieu scolaire en termes de \\
restauration (cantine...) \\
\hline Les services de restauration à proximité de l'école \\
(camions, lolos...) \\
\hline Les services de transport scolaire \\
\hline Les bâtiments de votre lieu d'étude (classe, cours de \\
récréations...) \\
\hline Les services de soutien à l'intégration scolaire et à \\
l'aménagement scolaire, égalité des chances (ASH) \\
\hline Les horaires scolaires \\
\hline Les associations de parents d'élèves ou associations de \\
collégiens, lycéens ou étudiants
\end{tabular}


Comme précédemment, le tableau 2 ci-dessous présente plus globalement les modifications quantitatives opérées sur la MQE, pour chacun des facteurs physiques et sociaux.

Tableau 2. Nombre d'items par domaine des versions originale et modifiée de la MQE

\begin{tabular}{|c|c|c|c|}
\hline Facteurs sociaux & nb items & Facteurs physiques & nb items \\
\hline Réseau social & $5^{*} 7^{* *}$ & Accessibilité physique & $7^{*}$ \\
\hline Attitudes de l'entourage & $9 * 18 * *$ & Sol, voies de circulation et distances & $10 * 2 * *$ \\
\hline Marché du travail & $8 * 3 * *$ & Éléments naturels & $9 * 9 * *$ \\
\hline Milieu professionnel ou de s & stage & Objets & $12^{*}$ \\
\hline $\begin{array}{l}\text { Services des infrastructures } \\
\text { publiques }\end{array}$ & $10^{*}$ & Technologie & $2^{*}$ \\
\hline Sources de revenus & $7^{*} 2^{*}$ & Aides techniques & $3 *$ \\
\hline Services commerciaux & $2^{*}$ & & \\
\hline Services juridiques & $1^{*}$ & & \\
\hline Services socio-sanitaires & $7 * \quad 8^{* *}$ & & \\
\hline Services éducatifs & $12^{*} 10^{* *}$ & & \\
\hline $\begin{array}{l}\text { Services des organismes } \\
\text { communautaires }\end{array}$ & $4 * \quad 4^{* *}$ & & \\
\hline Systèmes politiques & $6^{*} 3 * *$ & & \\
\hline Règles sociales & $4 * 3 * *$ & & \\
\hline
\end{tabular}

\section{Phase 2 : recueil de données de l'étude Sociodrep : objectifs et méthode}

II. 1. Axe quantitatif: évaluation de la réalisation des habitudes de vie et de la qualité perçue de l'environnement

La participation sociale et la qualité perçue de l'environnement sont respectivement évaluées à l'aide des instruments issus du modèle du PPH, la MHAVIE et la MQE, adaptés au contexte local. Lors de la passation de ces questionnaires, des informations complémentaires sont recueillies. Elles portent sur les variables socio-démographiques et les données cliniques suivantes : le lieu de résidence, le type d'établissement scolaire fréquenté, la profession des parents et leur situation maritale (en couple, séparés, parent veuf, famille recomposée) le 
nombre de frères et sœurs et la position dans la fratrie, et, pour l'année précédente (au cours des 12 derniers mois), la récurrence des crises, la survenue d'infections, et la fréquence des visites au Centre de la Drépanocytose. Pour chaque sujet anonymisé par l'attribution d'un code, toutes les données sont recueillies informatiquement et immédiatement stockées dans l'eCRF (Case Report Form) conformément aux règles éthiques validées par le Comité de Protection des Personnes (CPP).

Un groupe contrôle, composé d'enfants $(\mathrm{n}=45)$ sans maladie chronique a également été intégré à l'étude. Ce groupe témoin permet tout d'abord de fournir des données quant à la participation sociale, et plus précisément à la réalisation des habitudes de vie et à la perception de l'environnement des jeunes en Guadeloupe, afin de neutraliser un éventuel effet lié au contexte culturel spécifique. Les niveaux de la participation sociale et de la qualité perçue de l'environnement seront ainsi comparés entre jeunes malades et sujets sains (groupe contrôle) afin de s'assurer que les différences observées soient bien imputables à la pathologie et non au contexte culturel, les items spécifiques à la drépanocytose ayant été retirés lors de l'analyse comparative. D'autres travaux relatifs à la participation sociale, essentiellement dans le champ des incapacités et des déficiences, en contexte québécois, états-unien et européen ont ainsi procédé en utilisant un groupe témoin (e.g., Law et al., 2011 ; Sylvestre et al., 2013 ; Michelsen et al., 2014 pour une revue de la littérature). Notons qu'à ce jour, aucune étude n'a été produite pour évaluer la participation sociale dans le contexte spécifique guadeloupéen.

Ce groupe contrôle permet également de renforcer nos analyses puisque nous serons en mesure d'établir des comparaisons intergroupes (drépanocytaires vs non-malades), et plus seulement intragroupes (au sein du groupe drépanocytaire, entre sujets présentant des troubles neurocognitifs et individus sans trouble). Les analyses permettront ainsi d'appréhender l'effet de la drépanocytose en évaluant, de plus, la contribution éventuelle de troubles neurocognitifs : Comment l'expérience de la drépanocytose impacte la participation sociale des enfants et adolescents guadeloupéens ? Cet effet est-il modulé par la présence de difficultés neurocognitives ? Comment ces éléments interagissent-ils avec d'autres variables environnementales, dans une approche systémique ? Notre hypothèse principale prévoit que la participation sociale sera significativement plus faible chez les enfants et adolescents drépanocytaires, et ce d'autant plus qu'ils présentent des troubles cognitifs. De façon plus opérationnelle, on s'attend 1) à ce que les scores de réalisation des habitudes de vie (score global de participation sociale et sous-scores des dimensions de la MHAVIE) et de qualité perçue de l'environnement (score global, sous-scores liés à l'environnement physique, et social de la MQE) soient significativement plus faibles chez les sujets drépanocytaires. 2) Au sein de ce 
groupe, on fait l'hypothèse que les sujets présentant des difficultés d'ordre cognitif auront des scores plus faibles que les patients dont le fonctionnement cognitif est évalué comme « normal ». Les premiers traitements statistiques des données liées à la participation sociale et à la perception de l'environnement confirment la première hypothèse opérationnelle (article soumis).

L'utilisation conjointe de la MHAVIE et de la MQE est pertinente, puisqu'elle s'appuie sur le postulat de relations dialectiques entre l'évaluation de l'environnement et la réalisation des habitudes de vie d'une personne ou d'un groupe de personnes. Les outils sont complémentaires, car ils permettent d'obtenir une mesure de la qualité de la participation sociale des personnes vivant des situations de handicap, qualité fortement dépendante de son contexte, c'est-à-dire de la qualité de l'environnement tant social que physique (Boucher et al, 2015 ; Fougeyrollas et $a l ., 2015)$. Ainsi, la réalisation d'une activité quotidienne telle que le« déplacement » est en lien étroit avec la qualité de l'environnement, que ce soit sur le plan micro-personnel (domicile), méso-communautaire (quartier, réseau de transport) et macro-sociétal (politique d'aménagement et d'accessibilité, Boucher et al., 2017).

II.2. Axe qualitatif du projet : Appréhender la «carrière » drépanocytaire et le processus de production d'une maladie chronique

Notre recherche mobilise également une méthodologie qualitative, par le biais d'entretiens de type « récits de vie » qui sont conduits avec les jeunes drépanocytaires et leurs proches. Ce type d'entretiens, qualifiés de biographiques, ou d' « entretiens en profondeur » par Bogdan et Taylor (1975), vise à « la compréhension des perspectives des gens interviewés sur leur vie, leurs expériences ou leurs situations, et exprimées dans leur propre langage » (Lapassade, 1991 : 38). L'objectif de ce recueil de données, complémentaire du versant quantitatif, est de produire des entrées thématiques similaires à celui-ci afin d'approfondir chaque domaine de participation sociale et d'identifier les éléments biographiques et familiaux permettant de replacer ces données «désincarnées » dans un contexte, une temporalité, et surtout, une trajectoire de vie ${ }^{16}$. Ces différents entretiens permettront donc, après les analyses des questionnaires, d'approfondir la compréhension qualitative du vécu du patient et de sa famille. La méthode de recrutement ici retenue n'a pas pour vocation d'être significative sur le

\footnotetext{
${ }^{16}$ En parallèle, nous projetions de mener des observations sur le terrain médical et associatif (pratiques et interactions des praticiens avec les patients et au sein de l'équipe, suivi de séances d'éducation thérapeutique, structuration du milieu associatif), mais ceci n'a pu être réalisé en raison de contraintes temporelles et de résistances à l'observation sociologique des pratiques médicales dans un contexte sensible.
} 
plan statistique, objectif visé par une méthode d'échantillonnage aléatoire, mais d'être représentative d'une population socialement signifiante pour les acteurs (Ferez, 2000). Deux critères méthodologiques la contraignent : la nécessité d'assurer la diversité de l'échantillon et la clôture de l'enquête sur la base du principe de saturation (Bertaux, 1997). D'après Strauss et Corbin (1990), celle-ci est établie lorsque 1) les données recueillies n'apportent plus aucune nouvelle information ; 2) les catégories incluent tous les éléments du phénomène et ne sont plus enrichies par de nouvelles données ; 3) les liens entre les catégories sont établis et validés.

Chaque entretien, enregistré, est anonymisé, et retranscrit mot à mot (verbatim) puis fera l'objet d'une analyse thématique. La réalisation des entretiens biographiques a lieu à la suite de l'enquête quantitative (MHAVIE \& MQE). Si la passation de ces questionnaires se déroule généralement au sein du Centre de la Drépanocytose, le recueil qualitatif est plutôt proposé à domicile, l'enquêteur se déplaçant, selon la convenance de l'enquêté. Initialement, il s'agissait d'interroger les jeunes malades de façon isolée, mais ce projet fut difficilement envisageable. Lors des premières entrevues, nous ressentions chez les familles une crainte, essentiellement exprimée de façon informelle, à l'idée que la situation d'entretien pourrait susciter des confidences, des révélations, voire mettre au jour des discordes entre les membres de la famille, conduisant ainsi les proches et parfois les malades eux-mêmes à refuser d'être interrogés de façon isolée (pour des difficultés similaires dans le recueil de discours auprès de drépanocytaires, voir les travaux de Bougerol, 1994).

Au cours des récits de vie, plusieurs thèmes sont abordés : l'enfance, le parcours scolaire, le parcours familial (les parents, emploi, mariage, séparation...), la fratrie, l'implication dans les associations, les pratiques sportives, le vécu médical et des hospitalisations. Avec les parents, les aspects biographiques avant et après l'annonce de la maladie sont privilégiés, mais aussi les représentations sociales de celle-ci. Plusieurs axes de travail guideront l'analyse : on s'attachera à mettre en évidence la façon dont la drépanocytose génère des effets sur la participation sociale, en termes de facilitations ou de difficultés, puis à identifier les espaces d'exclusion ou d'auto-exclusion. En dernier lieu, seront décrits les effets générés par les facteurs environnementaux (e.g., famille, société, groupe d'affiliation, représentations sociales de la maladie).

Dans ce recueil, la considération de la dynamique temporelle est essentielle : dépourvue de perspective de guérison ${ }^{17}$, ou de rétablissement d'un état antérieur, la maladie chronique

\footnotetext{
${ }^{17}$ Il faut nuancer ce propos en précisant que depuis une vingtaine d'années, l'allogreffe de cellules souches hématopoḯtiques est pratiquée chez des patients drépanocytaires, à partir de donneurs intrafamiliaux (De
} 
demande à être gérée continuellement, à la différence de la maladie « aigüe », transitoire. Elle ne constitue donc pas un épisode singulier dans la vie d'un sujet, mais elle est un état permanent qui se confond avec la vie même (Baszanger, 1986 ; Pierret, 1997, 2004). Cette caractéristique - d'être un élément à long terme, voire permanent de la vie d'une personne - rend nécessaire une analyse sociologique de cette expérience insistant sur son cadre temporel (Bury, 1982, 1991). En premier lieu, l'annonce du diagnostic entraine, pour l'individu malade, une « rupture biographique » (Bury, 1982 ; Corbin \& Strauss, 1987). Les travaux sociologiques ont ainsi analysé l'impact d'un tel bouleversement sur le vécu du malade et sur ses interactions sociales (Strauss \& Glaser, 1975), à partir du concept interactionniste de « carrière ». Il est alors possible d'identifier une «trajectoire » caractérisée par une véritable organisation du travail élaborée depuis le diagnostic, puis tout au long de l'évolution de la maladie pour la contrôler. Ce travail implique les négociations, avec les divers acteurs, opérées par le malade, sa gestion quotidienne de la pathologie ainsi que les réaménagements identitaires et biographiques. Dans notre cas, la rupture biographique n'est pas tant éprouvée par les jeunes malades, «nés avec la maladie » et diagnostiqués à la naissance, que par les parents qui font face à un bouleversement de leur vie et de leur quotidien, excepté ceux qui avaient déjà en charge un enfant malade ou qui souffraient eux-mêmes de la drépanocytose. Cette rupture peut s'incarner dans de nouvelles limitations de leur participation sociale (par exemple, ne plus pouvoir pratiquer d'activités sportives en montagne, du fait de la faible oxygénation, ne plus se baigner en rivière en raison de la température de l'eau ou encore être contraint de quitter son emploi du fait des multiples hospitalisations de l'enfant), limitations parfois déjà existantes lorsque le parent est également drépanocytaire ou que l'enfant a un membre aîné de la fratrie qui est aussi porteur de la pathologie ${ }^{18}$.

Ainsi, resituer les données au sein d'une trajectoire, en considérant comme essentielle la temporalité, du fait du caractère chronique de la maladie, permet d'éclairer ce processus par une lecture non seulement synchronique, mais aussi diachronique des éléments en jeu, comme le suppose la dernière évolution du modèle du MDH-PPH2 (Fougeyrollas, 2010 ; Fougeyrollas, Boucher \& Charrier, 2016). En effet, la révision du PPH tend à rendre compte de l'expérience des personnes vivant leur processus de développement personnel comme une adaptation continue avec des potentiels de capacités singulières, qui ne subissent donc pas de « rupture » (en tant qu'entrée «brutale» dans le système, comme le spécifiait davantage le précédent

Montalembert, 2000 ; Bernaudin et al., 2004). Les pourcentages de survie sont élevés (entre 84 et 94\%, Ibid.), mais les complications sont possibles (toxicité, décès).

${ }^{18} \mathrm{Ces}$ informations sont recueillies et annotées lors de la passation des questionnaires et entretiens. 
modèle du PPH), mais dont le développement est influencé par leurs limitations et par le sens socialement attribué à leur différence. De fait, comme c'est le cas dans la drépanocytose, lorsque ces déficiences sont acquises dès la naissance, l'expérience fonctionnelle dite « limitée » de ces enfants est vécue comme ordinaire et ce sont les significations attribuées, et les représentations sociales portées par les environnements dans lesquels ils évoluent, qui nourrissent la conscience de leur singularité. C'est un processus d'interaction multifactoriel complexe qui est à l'œuvre du fait du caractère évolutif des maladies chroniques, produisant des fluctuations et des ajustements où les variables environnementales sont à la fois des facteurs de risque ou de protection mais toujours déterminants de la qualité de participation sociale (Fougeyrollas, Boucher \& Charrier, 2016).

Les éléments discursifs ainsi obtenus permettront également d'assoir la pertinence des modifications apportées aux outils utilisés pour le versant quantitatif, en vue de leur adaptation contextuelle et locale, et contribuer à attester de leur validité. Ainsi, les réponses obtenues aux questionnaires, rendant compte de la réalisation d'habitudes de vie, de perception de difficultés et de satisfaction liées à ces activités courantes et rôles sociaux, ainsi que de la perception des facteurs environnementaux, sont obtenues à un instant $\mathrm{T}$, qui est celui du recueil ${ }^{19}$. Or, ces modalités de réalisation, ces perceptions et attributions ont une histoire d'élaboration, de renforcement, de fragilisation éventuelle : seule l'enquête sociologique, appuyée par la méthode de l'entretien biographique, consistant à reconstituer les étapes d'une expérience, d'une trajectoire, permet d'objectiver cette histoire.

L'usage de méthodes mixtes dans notre recueil de données nous assure donc une lecture croisée des résultats, et permettra une triangulation des données, définie comme la comparaison de données obtenues par deux ou plusieurs méthodologies différentes, contribuant à accroitre la puissance de l'interprétation (Denzin, 1989 ; Bosisio \& Santiago-Delefosse, 2014).

Enfin, le choix de cette méthodologie qualitative s'inscrit dans la volonté de valoriser le courant narratif, c'est-à-dire la production de significations et la mise en récit de l'expérience de la maladie et du handicap (Ville, 2014). Ce courant, et plus globalement l'approche qualitative, vient en appui du paradigme émancipatoire et compréhensif du handicap et de la maladie, tel que valorisé par le modèle humaniste du MDH-PPH2 qui affirme que «les

\footnotetext{
${ }^{19} \mathrm{Il}$ est précisé dans les instructions pour la passation de la Mhavie, que « pour chacune des habitudes de vie, vous devez indiquer de quelle façon le jeune la réalise généralement, dans son quotidien. Il s'agit de la façon la plus habituelle pour le jeune de la réaliser. ». Cependant l'on sait que les événements récents, même exceptionnels et non habituels risquent d'influencer le répondant.
} 
méthodes interactionnistes sont essentielles à l'approche émancipatoire» (Boucher, 2003 : 122).

\section{CONCLUSION}

Théoriquement arrimé au modèle du MDH-PPH2 (Fougeyrollas, 2010 ; Fougeyrollas, Boucher \& Charrier, 2016), notre projet a pour objectif d'évaluer la participation sociale d'enfants et adolescents drépanocytaires guadeloupéens, présentant ou non des troubles neurocognitifs, et d'appréhender la façon dont le vécu de la maladie chronique, en lien avec des difficultés personnelles, et se déployant au sein de divers environnements physiques et sociaux, agit sur leurs réalisations quotidiennes et leurs rôles sociaux. Sur le plan empirique, les outils de ce modèle sont utilisés pour mesurer les habitudes de vie et la qualité perçue de l'environnement (MHAVIE 4.0 et MQE 2.0). L'usage conjoint de ces échelles, considérées comme complémentaires, est pertinent bien que non systématique dans la littérature (Boucher et al, 2015). Tenant compte de l'adaptation locale valorisée dans les travaux et actions de promotion des droits des personnes en situation de handicap (Boucher et al., 2017), nous avons opéré sur ces outils des ajustements afin de considérer les spécificités culturelles de la Guadeloupe mais aussi celles liées à la pathologie de notre population d'étude.

Nos choix méthodologiques et épistémologiques contribuent à enrichir la littérature sur la participation sociale, en apportant un éclairage multiple sur les processus en jeu dans la production de la maladie chronique et du handicap, plus particulièrement pour ces jeunes drépanocytaires vivant en Guadeloupe. Notre étude s'inscrit dans une démarche interdisciplinaire voire transdisciplinaire (cf. Charaudeau, 2010), à l'interface des travaux biomédicaux, neuropsychologiques et en sciences sociales. Nous mobilisons ainsi des méthodologies mixtes : quantitative avec l'utilisation des échelles MHAVIE et MQE, de tests neuropsychologiques afin d'évaluer le fonctionnement cognitif et la présence éventuelle de troubles, des données biomédicales, et qualitative, via la réalisation d'entretiens biographiques (récits de vie) avec les malades et leurs proches. Cet axe de recueil qualitatif, adossé à l'usage d'outils quantitatifs d'évaluation de la participation sociale, constitue un apport à la littérature existante sur la participation sociale et ouvre des perspectives quant à la compréhension de la réalisation des habitudes de vie et des rôles sociaux, au sein d'un modèle systémique complexe tenant compte des facteurs personnels et de la perception de l'environnement. Si le clivage épistémologique entre méthodes quantitatives et qualitatives, longtemps réputées inconciliables, est à présent dépassé (Creswell \& Clark, 2007 ; Pluye, 2012), notre « démarche 
de construction du savoir » préférable au terme de méthodologie (cf. Garric \& CapdevielleMougnibas, 2009 ; Bosisio \& Santiago-Delefosse, 2014), postule la nécessité de mobiliser des savoirs, méthodes et regards disciplinaires distincts mais complémentaires dans l'étude d'un objet transversal. Pour autant, la mobilisation, juxtaposition, mise en dialogue des disciplines et des paradigmes n'est pas dépourvue de tensions et demande un travail de négociation.

L'ambition de notre recherche est d'intégrer, dans les analyses finales, l'ensemble des données : biomédicales, neuropsychologiques et relatives à la participation sociale, complétées par les productions discursives issues des récits de vie. Lors de ces analyses sera valorisée, en fonction de la modélisation et hiérarchisation des facteurs impliqués, une approche restituant l'importance de la contribution environnementale et plus particulièrement sociale dans la production des obstacles à la participation sociale, éloignant la perspective biomédicale et essentialisante du handicap/de la maladie, en accord avec le cadre conceptuel du PPH (Ravaud \& Fougeyrollas, 2005). À cette étape du traitement des données, se pose avec davantage de force la question de la gestion et de l'opérationnalisation de l'inter-pluri-transdisciplinarité. Cette transdisciplinarité constitue donc l'une des richesses, mais aussi l'un des enjeux majeurs de notre projet de recherche, et cette réflexivité qui s'esquisse ici est, et sera à l'œuvre tout au long de ce travail (Ruffié et al., 2020).

Cette recherche ambitionne, au-delà de montrer la pertinence de la conceptualisation du PPH dans la compréhension des déterminants de la participation sociale chez des drépanocytaires, et de l'adaptation de ses outils de mesure à l'expérience de la maladie et au contexte guadeloupéen, de mener une réflexion épistémologique soulevée par un objet transversal. Cette pensée de la complémentarité nourrira les étapes qui jalonnent notre projet de recherche et contribuera à alimenter de vifs et actuels débats quant aux travaux pluridisciplinaires dans le champ de la santé (Eideliman \& Kivits, 2012 ; Guével \& Pommier, 2012 ; Trabal, 2019 ; Fresneau \& Gautier, 2020).

\section{Références}

Adeyemo, T. A., Ojewunmi, O.O., Diaku-Akinwumi, I.N., Ayinde, O.C. \&Akannu, A.S. (2015). Health related quality of life and perception of stigmatisation in adolescents living with sickle cell disease in Nigeria: A cross sectional study. Pediatric Blood Cancer, 62(7), 1245-51.

Atkin, K., \&Ahmad, W. (2001). Living a 'normal' life: young people coping with thalassaemia major or sickle cell disorder. Social Science \&Medicine, 53(56), 615-626. 
Bardakdjian J. \&Wajcman, H. (2004). Epidémiologie de la drépanocytose. Revue Pratique, $54(14), 1531-3$.

Baszanger, I. (1986). Les maladies chroniques et leur ordre négocié. Revue Française de Sociologie, 27, 3-27.

Bedell, G. \& Coster, W. (2008). Measuring participation of school-age children with traumatic injuries: Considerations and approches. Journal of Head Trauma Rehabilitation, $18(1), 65-82$.

Bediako, S. M. \& Moffitt, K. R. (2011). Race and Social Attitudes about Sickle Cell Disease. Ethnicity and Health, 16(4-5), 423-429.

Benjamin, L. (2008). Pain management in sickle cell disease: Palliative care begins at birth? ASH Education Program Book, 1, 466-474.

Benton, T.D., Boyd, R., Ifeagwu, J., Feldtmose, E. \& Whitley, K. S. (2011). Psychiatric diagnosis in adolescents with sickle cell disease: A Preliminary Report. Current Psychiatric Report, 13(2), 111-5.

Berkelhammer, L. D., Williamson, A. L. Sanford, S. D., Dirksen, C. L., Sharp, W. G., Margulies, A. S. \& Prengler, R. A. (2007). Neurocognitive sequelae of pediatric sickle cell disease: A review of the literature. Child neuropsychology, 13(2), 120-31.

Bernaudin, F., Pradère, J., Taïeb, O., Abbal, T., Champion, M., Dutray, B. ... Moro, M. R. (2004), Perspectives thérapeutiques, cliniques et psychologiques dans les formes sévères de drépanocytose, In : Lainé A., (dir), La Drépanocytose. Regards croisés sur une maladie orpheline, Paris, Karthala, 376 p.

Bertaux, D. (1997). Les récits de vie. Paris: Nathan.

Bibrac, A. \& Etienne-Julan, M. (2004). Le centre caribéen de la drépanocytose « Guy Mérault » : un modèle de prise en charge globale et intégré. In : Lainé A., (dir.), La Drépanocytose. Regards croisés sur une maladie orpheline, Paris, Karthala, 376 p. 
Bogdan, R. \& Taylor, S. J. (1975). Introduction to qualitative Research methods. A phenomenological Approach to the social Sciences. New York: London John Wiley and Sons.

Boisvert, Y. (2006). Modèle clinique d'application du cadre conceptuel du Processus de production du handicap auprès de personnes présentant des incapacités intellectuelles. In Gascon H., Boisvert D., Haelewick M-C., Roulin J-R. et Detraux J-J. Déficience intellectuelle : savoirs et perspectives d'action. Tome 1 : Représentations, diversités, partenariat et qualité. Québec : Les Éditions Presses Inter Universitaires, 31-41.

Bonnet, D. (2001). Rupture d'alliance contre rupture de filiation. Le cas de la drépanocytose en Côte d'Ivoire. In : Dozon J.P. et Fassin D. (dir.) La Culture selon la santé publique. Paris : Balland, $332 \mathrm{p}$.

Bosisio, F. \& Santiago-Delefosse, M. (2014). Intérêts et limites de l'utilisation d'une méthodologie mixte: à propos d'une recherche en psychologie de la santé. Pratiques psychologiques, 20(1), 39-53.

Boucher, N. (2003). Handicap, recherche et changement social. L'émergence du paradigme émancipatoire dans l'étude de l'exclusion sociale des personnes handicapées. Lien social et Politiques, 50, 147-164.

Boucher, N., Beaudoin, R., Fougeyrollas, P., Hazard, D. \& Vincent, P. (2017). Guide méthodologique pour la planification et l'évaluation des actions de promotion des droits des personnes en situation de handicap. Édition novembre 2017, Bibliothèque nationale du Québec.

Boucher, N., Fiset, D., Lachapelle, Y. \& Fougeyrollas, P. (2013). Projet d'expérimentation de la MHAVIE en sites-pilotes des centres de réadaptation en déficience physique et intellectuelle, troubles envahissant du développement et des centres de santé et services sociaux, volet soutien à domicile. Rapport final CIRRIS-IRDPQ. 
Boucher, N., Vincent, P., Geiser, P. \& Fougeyrollas, P. (2015). Participation des personnes en situation de handicap à la gouvernance locale : présentation d'un projet visant à mesurer l'impact des stratégies de développement local inclusif. Alter, European Journal of Disability Research 9(1), 51-63.

Bougerol, C. (1994). Approche anthropologique de la drépanocytose chez des malades antillais. Sciences sociales et santé, 12(3), 48-68.

Boukala, M. (2009). Entretien avec Patrick Fougeyrollas. La nouvelle revue de l'adaptation et de la scolarisation,45(1), 165-174.

Bourdieu, P. (1980). Le Sens pratique. Paris: Ed. de Minuit.

Bulgin, D., Tanabe, P.\&Jenerette, C. (2018). Stigma of Sickle Cell Disease: A Systematic Review. Issues in Mental Health Nursing, 39(8), 675-686.

Bury, M. (1982). Chronic illness as a biographical disruption. Sociology of Health and Illness, 4, 167-182.

Bury, M. (1991). The sociology of chronic illness: a review of research and prospects. Sociology of Health and Illness, 13, 451-468.

Caballero, F.F, Miret, M., Power, M., Chatterji, S., Tobiasz-Adamczyk, B, Koskinen, S.... Ayuso-Mateos, J. L. (2013). Validation of an instrument to evaluate quality of life in the aging population: WHOQOL-AGE. Health and Quality of Life Outcomes, 11(177), 2-12.

Carpentier, M.Y., Elkin, T.D. \& Starnes, S.E. (2009). Behavioral inhibition and its relation to anxiety and depression symptoms in adolescents with sickle cell disease: A preliminary study. Journal of Pediatric Oncology Nursing, 26(3), 156-66.

Charaudeau, P. (2010). Pour une interdisciplinarité « focalisée » dans les sciences humaines et sociales. Questions de Communication, 17, 195-222. 
Corbin, J. \& Strauss, A. (1987). Accompaniments of Chronic Illness: Changes in Body, Self, Biography and Biographical Time. Research in the Sociology of Health Care, 6, 249281.

Creswell, J., \& Plano Clark, V. (2007). Designing and Conducting Mixed Methods Research. Thousand Oaks, CA: Sage.

De Montalembert, M. (2000). Actualités sur le traitement de la drépanocytose. Journal de Pédiatrie et de Puériculture, 13, 206-210.

Denzin, R. (1989). The Research Act: A Theoretical Introduction to Sociological Methods. Englewood Cliffs NJ, Prentice Hall.

Desrosiers, J., Noreau, L., Rochette, A., Bravo, G. \& Boutin, C. (2002). Predictors of handicap situations following post-stroke rehabilitation. Disability and Rehabilitation, 24(15), $774-785$

Donkervoort, M., Roebroeck, M., Wiegerink, D., van der Heijden-Maessen, H. \& Stam, H. (2007). Transition Research Group South West Netherlands Determinants of functioning of adolescents and young adults with cerebral palsy. Disability and Rehabilitation, 29, 453-463.

Doré, S. (2007). D’ « À part... égale » à « Levons les obstacles » : 20 ans d'influence du modèle québécois du Processus de production du handicap. Développement humain, handicap et changement social, 16(1), 5-13.

Dumont, C. (2003). L'identification des facteurs qui vont favoriser la participation soiale des adultes présentant des séquelles de traumatisme cranio-cérébral. Thèse de doctorat. Université Laval.

Dyson, S. (2019). Sickle Cell and the Social Sciences:Health, Racism and Disablement. Routledge. 
Eideliman, J. S. \&Kivits, J. (2012). Dossier «Recherches en santé et santé de la recherche en sociologie - Orientations, professionnalisation, financements », Socio-logos. Revue de l'association française de sociologie, 7, mis en ligne le 30 octobre 2012, URL : http://socio-logos.revues.org/2679

Etienne-Julan, M. \& Saint-Martin, A. (2005). La drépanocytose aux Antilles Françaises. Revue francophone des laboratoires, 374, 61-66.

Ferez, S. (2000). De l'expression corporelle aux activités physiques artistiques : androcentrisme de l'E.P.S. et détournement d'une lutte. Corps et culture, 5, mis en ligne le 15 octobre 2007.

Ferez, S., Wallach, I., Gaucher, C., Héas, S., Ruffié, S., Thomas, J. \& Gaissad, L. (2015). Effets du diagnostic d'infection au VIH sur la participation sociale : enjeux de l'application du Processus de Production du Handicap à une maladie chronique. Développement humain, handicap et changement social, 21(2), 75-88.

Fougeyrollas, P. (1983). Entre peaux : logis de la différence. Du handicap à l'autonomie. Thèse de maitrise. Département d'anthropologie. Université Laval.

Fougeyrollas, P. (2010). La Funambule, le fil et la toile. Transformations réciproques du sens du handicap. Québec, Presses Universitaires de Laval.

Fougeyrollas, P., Bergeron, H., Cloutier, R., Côté, J. \& St-Michel, G. (1998). Classification québécoise : Processus de production du handicap. Québec, Réseau international sur le processus de production du handicap, RIPPH/SCCIDIH, 166p.

Fougeyrollas, P. \& Blouin, M. (1989). Handicaps et technologies. Anthropologie et Sociétés, 13(2), 103-113.

Fougeyrollas, P., Boucher, N. \& Charrier, F. (2016). Un modèle à l'épreuve du temps : le point sur le Modèle de développement humain et du Processus de production du handicap (MDH-PPH 2). Déficience intellectuelle et trouble du spectre de l'autisme, 8(1), 6-13. 
Fougeyrollas, P. \& Charrier, F. (2013). Modèle du Processus de production du handicap. EMC -Kinésithérapie-Médecine physique-Réadaptation, 9(3), 1-8.

Fougeyrollas, P., Fiset, D., Boucher, N., Grenier, Y., Gascon, H., Morales, E., Philibert, M. \& Charrier, F. (2015). Handicap, environnement et droits humains. Du concept d'accès à sa mesure. Développement humain, handicap et changement social. Numéro horssérie. Collection Espace Participation sociale. RIPPH.

Fougeyrollas, P., Lepage, C., Boissières, L.,Deaudelin, G. \& Doré, L. (2014). Assessment of Social participation in Three Measurement Times in Children with Traumatic Brain Injuries (TBI) Based on Parental Perceptions. Open Journal of Therapy and Rehabilitation, 2, 156-165.

Fougeyrollas, P., Noreau, L., Bergeron, H., Cloutier, R., Dion, S.-A. \& St-Michel, G. (1998). Social consequences of long term impairments and disabilities: conceptual approach and assessment of handicap. International Journal of Rehabilitation Research, 21, 127-141.

Fougeyrollas, P., Noreau, L., St-Michel, G. \&Boschen, K. (1999). Mesure de la Qualité de l'Environnement, version 2.0. RIPPH - C.P. 225, Lac St-Charles (Québec).

Fougeyrollas, P., Noreau, L. \& Lepage, C.(2014). La Mesure des habitudes de vie (MHAVIE 4.0). Version pour l'enfance de 5 à 13 ans. Québec, Réseau international sur le Processus de production du handicap (RIPPH).

Fresneau, J. \& Gautier, L. (2020). À la croisée des chemins : le jeune chercheur entre désir d'interdisciplinarité et ancrage monodisciplinaire. Encyclo. Revue de l'école doctorale Science dessociétés (ED 624), Université de Paris, 9-15.

Fucharoen, S. \&Winichagoon, P. (2000). Hemoglobinopathies in Southeast Asia: molecular biology and clinical medicine. CurrOpinHematol, 7(2), 106-112. 
Galactéros, F. (2005). Le diagnostic prénatal de la drépanocytose (Commentaire). Sciences sociales et santé, 23(2), 67-73.

Garric, N. \& Capdevielle-Mougnibas, V. (2009). La variation comme principe d'exploration des corpus de texte issus d'entretiens : intérêts et limites de l'analyse lexicométrique interdisciplinaire pour l'étude de la matérialité du discours. Corpus, 8, 105-128.

Gioia, G. A., Isquith, P. K., Guy, S. C. \& Kenworthy, L. (2000). Test review: Behavior Rating inventory of Executive Fonction. Child Neuropsychology, 3, 235-238.

Grandisson, M., Tétreault, S. \&Freeman, A. R. (2010). Le sport : promoteur de la santé et de la participation sociale en déficience intellectuelle. Revue Francophone de la déficience intellectuelle, 21, 54-65.

Graves, J. K.,Hodge, C. \&Jacob, E. (2016). Depression, anxiety, and quality of life in children and adolescents with sickle cell disease. Pediatric Nursing, 42(3), 113-9.

Groulx, L. H. (1997). Querelle autour des méthodes. Socio-anthropologie (en ligne). 2. Consulté à l'adresse : http://socio-anthropologie.revues.org/index30.html

Guével, M. R. \& Pommier, J. (2012). Recherche par les méthodes mixtes en santé publique : enjeux et illustration. Santé Publique, 1(24), 23-38.

HAS Haute Autorité de Santé - Syndromes drépanocytaires majeurs de l'enfant et de l'adolescent -Protocole national de diagnostic et de soins pour une maladie rare actualisé en 2014.

http://www.hassante.fr/portail/upload/docs/application/pdf/201004/ald10pndsdrepanoe nfantweb.pdf

Korkman, M., Kirk, U. \& Kemp, S. (2007). Nepsy-second Edition (NEPSY II). San Antonio, TX:Psychological Corporation.

Lachapelle, P. (2003). Processus de développement personnel et de production du handicap. Développement humain, handicap et changement social, 12(1), 50-54. 
Lacroix, N., Boucher, N. \& Villeneuve, P. (2016). Participation sociale et sclérose en plaques :

Réalité particulière. Service social, 62(2), 94-113.

Lainé, A. (dir.) (2004). La drépanocytose : regards croisés sur une maladie orpheline. Paris :

Karthala.

Lainé, A. \&Tchernia, G. (2010). « L'émergence » d'une maladie multimillénaire. Circulations de savoir et production d'inégalités face à la drépanocytose. In V. Chasles. Santé et mondialisation (pp. 239-261). Lyon: Université Jean-Moulin-Lyon 3.

Lapassade, G. (1991). L’ethnosociologie. Paris: Méridiens-Klincksieck.

Larivière, N. (2012). Comparaison de la participation sociale entre trois groupes de personnes ayant des troubles mentaux. Revue Développement humain, handicap et changement social, 20(2), 105-118.

Law, M., Anaby, D., Teplicky, R., AlunkalKhetani, M., Coster, W. \& Bedell, G. (2013) Participation in the home environment among children and youth with and without disabilities. British Journal of Occupational Therapy, 76(2), 1-9.

Lepage, C., Noreau, L. \& Bernard, P. (1998). Association between characteristics of locomotion and accomplishment of life habits in children with cerebral palsy. Physical Therapy, $78(5), 458-469$.

Luboya, E., Bousaka, J. C., Bothale Ekila, M. \&NtetaniAloni, M. (2014).Répercussions psychosociales de la drépanocytose sur les parents d'enfants vivant à Kinshasa, République Démocratique du Congo: une étude qualitative. Pan African Medical Journal, 19(5), 563-67.

Ludwig, N. N., Soumitr, S., Khowaja, M. K., Cohen, L.L, \&Dampier, C. (2018). Executive Functioning Mediates the Relationship Between Pain Coping and Quality of Life in Youth With Sickle Cell Disease. Journal of Pediatric Psychology, 43(10), 1160-1169.

Mauss, M. (1936). Les techniques du corps. Journal de Psychologie, XXXII, 3-4. 
Matos, M.A., Malheiros, C.D. \& Matos, S.R. (2016). Health Related Quality of Life of Patients with Sickle Cell Disease. Journal of Blood Disorder Transfusion, 7(345), 377-85.

Meremikwu, M. \&Okomo, U. (2011). Sickle cell disease. Clinical Evidence, 2011, 1-19.

Michelsen, S., Flachs, E. M., Damsgaard, M. T, Parkes, J., Parkinson, M., Rapp, M. ... Uldall, P. (2014). European study of frequency of participation of adolescents with and without cerebralpalsy. European journal of paediatric neurology, 18, 282-294.

Ninot, G., Soyez, F., Fiocco, S., Nassi, K., Morin, A. J. S., Prefaut, D. (2011). Le VQ11, un questionnaire de qualité de vie spécifique à la BPCO utilisable en clinique. Revue des maladies respiratoires, 27(5), 472-48.

Noreau, L. (2005). Un regard sur le passé pour mieux avancer vers l'avenir : La contribution du modèle conceptuel du Processus de production du handicap au développement de la recherche, Développement humain, handicap et changement social, 14(1), 28-35.

Noreau, L., Desrosiers, J., Robichaud L., Fougeyrollas, P., Rochette, A. \& Viscogliosi, C. (2004). Measuring social participation: Reliability of the LIFE-H in older adults with disabilities. Disability and Rehabilitation, 26, 346-352.

Organisation Mondiale de la Santé (2001). Classification internationale du fonctionnement, du handicap et de la santé. Genève, OMS.

Oudin-Doglioni, D., Gay, M.C., Lehougre, M.P., Arlet, J.B. \&Galactéros, F. (2019). Les représentations de la drépanocytose comme déterminants de l'observance thérapeutique. Annales Médico-psychologiques, revue psychiatrique,177(6), 517-525.

Pandian, V., Thompson, C. B., Feller-Kopman, D.J.,Mirski, M. A. (2015). Development and validation of a quality of life questionnaire for mechanically ventilated ICU patients. Crit Care Med, 43, 142-8.

Paré, C., Fougeyrollas, P., Parent, G. \& St-Michel, G. (2000). Participation sociale dans les habitudes de vie et identification des obstacles et des facilitateurs dans 
l'environnement de personnes présentant des limitations associées à un déficit intellectuel. Revue francophone de la déficience intellectuelle, 11(1), 27-41.

Parsh, B. \& Kumar, D. (2012). Understanding sickle cell disease. Nursing, 42(8), 65.

Piel, F.B, Patil, A.P., Howes, R.E., Nyangiri, O.A., Gething, P.W., Dewi, M. et al. (2013). Global epidemiology of sickle haemoglobin in neonates: a contemporary geostatistical model-based map and population estimates. Lancet, 381(9861), 142-151.

Pierret, J. (2004). Analyse dynamique de la mise sous multithérapie dans la cohorte française APROCO (1998-2000). In : Lévy J., Pierret J., Trottier G., eds, Les traitements antirétroviraux. Expériences et défis, Québec, Presses de l’Université du Québec, pp. $61-93$.

Pierret, J. (1997). Un objet pour la sociologie de la maladie chronique : la situation de séropositivité au VIH ? Sciences Sociales et Santé, 15(4), 97-120.

Pluye,P. (2012). Les méthodes mixtes. In Ridde, V.\& Dagenais, C. (eds.), Approches et pratiques en évaluation de programme (pp. 125-143).

Poku, BA, Caress, A-L, Kirk S. (2020). "Body as a Machine": How Adolescents With Sickle Cell Disease Construct Their Fatigue Experiences. Qualitative Health Research. 30(9), 1431-1444.

Préau, M., Protopopescu, C., Spire, B., Dellamonica, P., Poizot-Martin, I., Villes, V. ...

Carrierri, M. P. (2006). La qualité de vie des patients co-infectés par le VIH et le VHC. Revue d'Epidémiologie et de Santé Publique, 54(1), 33-43.

Pruneau, J., Ferez S., Maillard F., Philippon B. \& Hue O. (2009). Accès à la culture biomédicale et enjeux socio-symboliques des représentations de la drépanocytose dans une population scolaire de Guadeloupe. Etudes caribéennes, 12, [En ligne], mis en ligne le 9 septembre, http://etudescaribeennes.revues.org/document3684.html. 
Pruneau, J., Philippon, B., Maillard, F., \& Hue, O. (2008). Sport et drépanocytose : le paradoxe dans l'itinéraire thérapeutique des adolescents drépanocytaires « SS » en Guadeloupe. Sciences sociales et santé, 26, 2, 5-32.

Ravaud, J.F. \& Fougeyrollas, P. (2005). La convergence progressive des positions francoquébécoises. Santé, Société et Solidarité, 2, 13-27.

Ravaud, J.-F., Letourmy, A. \& Ville, I. (2002). Les méthodes de délimitation de la population handicapée, l'approche de l'Enquête Insee, "Vie quotidienne et santé”. Population, 3, $543-565$.

Rey, A. (1959). Test de copie et de reproduction de mémoire de figures géométriques complexes. Paris : ECPA.

Ruffié1, S. \& Cholley-Gomez ${ }^{1}$, M., Villoing, G., Ferez, S., Boucher, N., Fougeyrollas, P. (2020, in press). Penser les effets du désavantage social liés à la maladie chronique : enjeux épistémologiques d'un projet transdisciplinaire sur des jeunes drépanocytaires en Guadeloupe, Alter, European Journal of Disability Research. https://doi.org/10.1016/j.alter.2020.07.003

Saint-Martin, C., Romana, M., Bibrac, A., Brudey, K., Tarer, V., Divialle-Doumdo, L., Petras, ... Etienne-Julan M. (2013). Universal newborn screening for haemoglobinopathies in Guadeloupe (French West Indies): a 27-year experience. Journal of Medicine Screen, 20(4), 177-82.

Smaldone, A., Manwani, D., \& Green, N. S. (2019). Greater number of perceived barriers to hydroxyurea associated with poorer health-related quality of life in youth with sickle cell disease. Pediatric Blood \& Cancer, 66(7), e27740.

Steen, R.G., Fineberg-Buchner, C., Hankins, G, Weiss L, Prifitera A. \& Mulhern RK. (2005). Cognitive deficits in children with sickle cell disease. Journal of Child Neurology, 20(2), 102-7. 
Strauss, A., \& Corbin, J. (1990). Basics of qualitative research: Techniques and procedures for developing grounded theory. Newbury Park, CA: Sage.

Strauss, A. \& Glaser, B. (1975). Chronic illness and quality of life. St Louis: Mosby.

Sundd, P., Gladwin, M. T., Novelli, E. M. (2019). Pathophysiology of Sickle Cell Disease. Annual Review of Pathology: Mechanisms of Disease, 14(1), 263-292

Sylvestre, A., Nadeau, L., Charron, L., Larose, N.f\& Lepage, C. (2013). Social participation by children with developmental coordination disorder compared to their peers. Disability and Rehabilitation, 35, 1814-20.

Szymanski, I. O., Odgren, P. R., Fortier, N. L., Snyder, L. M. (1980). Red blood cell associated IgG in normal and pathologic states. Blood, 55, 48-54.

Thomas, V. J. \& Taylor, L. M. (2002). The psychosocial experience of people with sickle cell disease and its impact on quality of life: Qualitative findings from focus groups. British Journal of Health Psychology, 7(3), 345-363.

Trabal, P. (2019). De l'interdisciplinarité dans la recherche sur des dossiers sanitaires. Revue D’Epidemiologie Et De Sante Publique, 67.

Trabal, P., Collinet, C. \&Terral, P. (2017). Faire preuve d'interdisciplinarité : Un mot d'ordre, ses interprétations et ses ajustements. Terrains \& travaux, 30(1), 209-229.

Vickrey, B.G., Hays, R.D., Harooni, R., Myers, L.W. \& Ellison, G.W. (1995). A healthrelated quality of life measure for multiple sclerosis. Quality of Life Research, 4(3), 187-206.

Ville, I. (2014). Les savoirs de la sociologie. Dans : Charles Gardou éd., Handicap, une encyclopédie des savoirs (pp. 399-413). Toulouse, France: ERES.

Wechsler, D. (2005). Wechsler Intelligence Scale for Children-Fourth Edition Spanish. San Antonio, TX:Psychological Corporation. 